\title{
Enhanced Mobility with Connectivity and Automation: A Review of Shared Autonomous Vehicle Systems
}

\author{
Liuhui Zhao, Member, IEEE, Andreas A. Malikopoulos, Senior Member, IEEE
}

\begin{abstract}
Shared mobility can provide access to transportation on a custom basis without vehicle ownership. The advent of connected and automated vehicle technologies can further enhance the potential benefits of shared mobility systems. Although the implications of a system with shared autonomous vehicles have been investigated, the research reported in the literature has exhibited contradictory outcomes. In this paper, we present a summary of the research efforts in shared autonomous vehicle systems that have been reported in the literature to date and discuss potential future research directions.
\end{abstract}

Index Terms-Shared mobility, carsharing, connected and automated vehicles

\section{INTRODUCTION}

\section{A. Movitation}

In a rapidly urbanizing world, we need to make fundamental transformations in how we use and access transportation. We are currently witnessing an increasing integration of our energy and transportation which, coupled with the human interactions, is giving rise to a new level of complexity [1] in emerging transportation systems such as connected and automated vehicles (CAVs) and shared mobility. As we move to increasingly complex emerging transportation systems, new control approaches [2], [3] are needed to optimize their impact on the mobility system behavior.

Shared mobility includes a variety of service models (e.g., carsharing, ridesharing, bikesharing) to meet travel needs and may result in a transformative impact on urban mobility [4][8] and landscape. As shared mobility services evolve, there has been a debate on their potential impact [7], [9], [10]. The advent of intelligent transportation systems and information technologies has aimed at facilitating shared mobility services (Fig. 1). In this context, impact analysis of the introduction of connected vehicles and automated vehicles (AVs) into existing shared mobility services is vital to identify the opportunities and challenges related to a shared autonomous mobility system. In this paper, we review the research reported in the literature on carsharing enhanced by vehicle connectivity and automation technologies, i.e., shared autonomous vehicle (SAV) system, and discuss potential implications in the environment and urban mobility.

This research was supported in part by ARPAE's NEXTCAR program under the award number DE-AR0000796 and by the Delaware Energy Institute (DEI).

The authors are with Department of Mechanical Engineering, University of Delaware, Newark, DE 30332; e-mails: (lhzhao@ieee.org, andreas@udel.edu).

\section{B. Background}

There are different types of carsharing service models, including round-trip carsharing, one-way station-based or freefloating carsharing, and peer-to-peer carsharing [5], [11]. In the past few years, short-term vehicle rental services provided by carsharing companies in major cities has attracted millions of users, while the number is expected to grow significantly [9], [10], [12]. Generally, it is believed that carsharing has positive impacts on energy use and greenhouse gas (GHG) emissions [12]-[16], particularly when low-polluting vehicles are introduced into the transportation systems [17]. Although there is evidence that the use of carsharing services leads to a decrease in vehicle ownership [12]-[14], location-specific variations (e.g., urban form, level of transit service, availability of alternative modes, etc.) has an impact on vehicle miles traveled (VMT) and public transit ridership [9], [10], [12], [13], [18].

The emerging CAV technologies offer intriguing opportunities to enhance urban mobility and traffic safety, and the introduction of CAVs enables innovative often more responsive and efficient options for traveling which may change the way people use mobility services [19], [20]. It is likely that the wide adoption of CAVs could also affect the usage of existing infrastructure to better serve the ever-changing transportation network [21]. While the benefits of CAV technologies on traffic flow and safety [22]-[26], coordination in specific traffic scenarios [27]-[33], and energy improvement on vehicle level

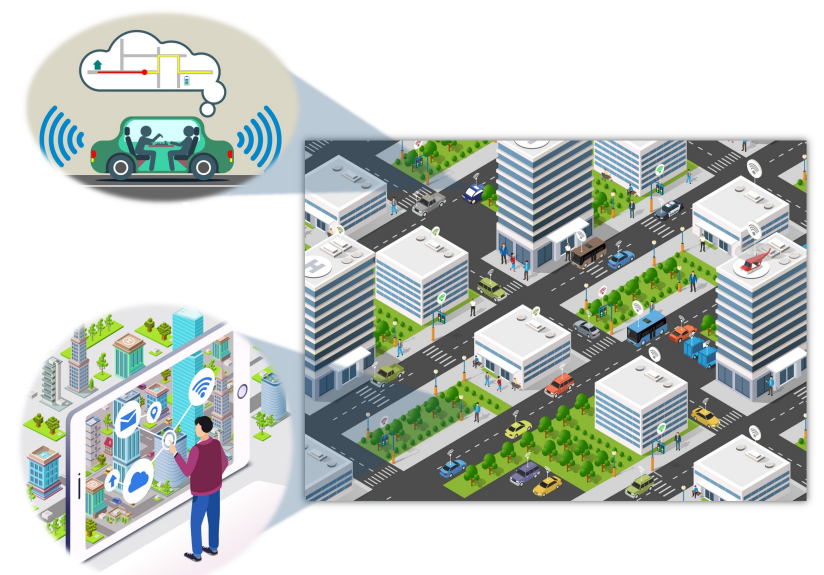

Fig. 1: A view of a city enhanced by connectivity and automation. 
[34]-[36] are well understood, potential deployment of the $\mathrm{CAV}$ s for the shared mobility service has raised a number of key questions related to fleet sizing, operation strategies and the implications on mobility, urban form, and environment [37]-[41].

With the ongoing growth of shared mobility and increasing interests in CAV fleet, the convergence of emerging mobility service and technology is still evolving. Many major automakers and technology companies are launching SAV pilot projects in the US and around the world, e.g., Ford, Voyage, Waymo, Uber, and Lyft [42]. While there is currently no largescale deployment of SAV fleet, several research efforts have evaluated the impacts of the SAVs, including simulation-based evaluation on environmental impact, cost-benefit, or demand analysis, e.g., [43]-[57]. There has been much contention on the potential influence of SAVs on travel behavior, urban landscape, congestion, and environment [58]. Although it seems that the required fleet size as well as the parking spaces to meet existing travel demand might drop significantly, multiple studies have indicated that full automation is likely to induce travel demand and attract new user groups, which may result in a potential increase in energy consumption, e.g., [59], [60]. Furthermore, there have been also concerns that SAVs might attract considerable attention from public transit patrons rather than private car owners, with implications on escalating traffic congestion, if not properly managed, e.g., [61], [62].

\section{Scope of the Paper}

In this paper, we review research efforts on the modeling and operations of the SAV system and try to identify potential research gaps that require further investigation. In our review, we have excluded studies on the demand estimation and travel behavior analysis of the SAVs. We applied the following search strings and included the papers up to date containing any combination of the keywords in the title, abstract, or keywords:

1) Shared autonomous (electric) vehicles, shared automated vehicles, autonomous vehicle sharing;

2) Autonomous carsharing, driverless carsharing, selfdriving carsharing;

3) Autonomous taxi, automated taxi, driverless taxi;

4) Automated demand responsive transport, autonomous mobility on demand, automated mobility on demand, autonomous mobility as a service.

Although the exploration of benefits of SAVs is still in early stages, we note that there are many aspects in common with the conventional carsharing system (with or without the option of ridesharing). There are several review papers providing a good summary under the umbrella of shared mobility, e.g., see [63]-[69]. Similar review efforts on the SAVs include the study by Hao and Yamamoto [70], who focused on the features and demand aspects of the SAV system through examining the corresponding aspects of car sharing in AVs. The most recent work conducted by Stocker and Shaheen [42] reviewed SAV pilots and legislation in the US, and discussed current and future development of the SAV system. Any such effort has obvious limitations. Space constraints limit the description of each paper in details, and thus, discussions are included only where they are important for understanding the fundamental concepts or explaining significant departures from previous work.

\section{Organization of the Paper}

The structure of the paper is organized as follows. In Section II. we present an overview of the SAV system and modeling approaches that have been commonly adopted. We then identify major design variables and system operating parameters that are widely studied in the literature to date and summarize the research efforts in Section III], including the problems of fleet sizing, vehicle assignment and relocation, consideration of electric vehicles, and ridesharing. In Section IV] we discuss different operation schemes of the SAVs in a mixed traffic environment that have been investigated in the literature. Finally, we discuss research gaps and potential future research directions in Section $\nabla$

\section{Shared AUtonomous VehiCLE SyStem MOdELING}

SAVs provide carsharing with a way of seamlessly relocating vehicles to better match dynamic demand [46]. As pilot programs of SAVs are beginning to accelerate around the world, there has been an increasing interest in investigating the SAV system. In this section, we first introduce earlier work on the feasibility of statewide implementation of SAVs and system performance analysis along with the cost-benefit analysis. We then discuss two major directions in modeling and analysis of the SAV system: (1) the development of analytical models along with specific problems that include vehicle assignment and rebalancing, e.g., [71]-[74]; (2) the development of agent-based models to emphasize the understanding of system performance and impact of the SAV system under different scenarios with a variety of parameters settings, e.g., [61], [75]-[79].

1) Feasibility analysis: In an early work [43], Ford proposed a statewide SAV system in New Jersey with a gridbased network model. The author discussed different operation strategies of a SAV system at different time periods. For example, in rush hours, the SAVs would function like a personal rapid transit (PRT) system to satisfy travel demand and ease congestion, whereas during non-rush hours, the SAVs could be operated with more flexibility and provide door-todoor service. The area considered in the paper was modeled as gridded zones, where a fixed SAV station would be located at the center of each cell. Later, Brownell and Kornhauser [80] described in detail two distinct SAV network models, i.e., PRT and the smart paratransit (SPT), and discussed the feasibility of a statewide SAV network in New Jersey. In the PRT network, fixed stations of the SAV system are established and passengers need to walk to their closest stations. Ridesharing is considered only if two passengers share the same origindestination pair and arrive at the station within a predefined time window. The idea behind the SPT system is that trips with close origins and/or destination will be served by one single vehicle. The vehicle moves around within the origin cell to pick up multiple passengers before traveling to the 
destination cell. Along the ways, the vehicle may stop at one, or more, locations to pick up or drop off passengers. In a SPT system with AVs, since the vehicle takes the place of the individual for accessing service, the distance between nodes in the transit grid could be increased. Burns et al. [44] conducted a cost-benefit analysis of a SAV system where the entire trip demand is satisfied by SAVs. To estimate the performance of a SAV system and compare with other systems (e.g., personal vehicle), the authors developed an analytical model with spatial queueing approach based on simplifying assumptions (e.g., uniformly distributed origins and destinations, constant trip request rate, etc). The results from three case studies showed that a SAV system is capable of providing better mobility experience at a significantly lower cost, in addition to its environmental and safety benefits.

2) Analytical modeling: Several research efforts reported in the literature have treated a SAV system as a spatial queueing system where passengers arrive at each station, pick up the vehicles - if parked at the station - and wait or leave the system, if no vehicle is available (Fig. 2). After dropping off passengers at their destinations, vehicles either start the next service, or park, or relocate themselves to other stations, e.g., [71]-[74], [81], [82]. For instance, Zhang et al. [38] described a SAV network as a spatial queueing system where transportation requests queue up and are served by the SAVs in the network. The authors presented two models for SAV systems. In the first model, the authors considered a distributed approach, where the objective is to design a routing policy that minimizes the average steady-state time delay between the generation of an origin-destination pair and the time the trip is completed. In the second model, the authors considered a lumped approach - customers are assumed to arrive at a set of stations in the network, where each customer picks up a vehicle, if available, or leaves the system, if no vehicle is parked at the station.

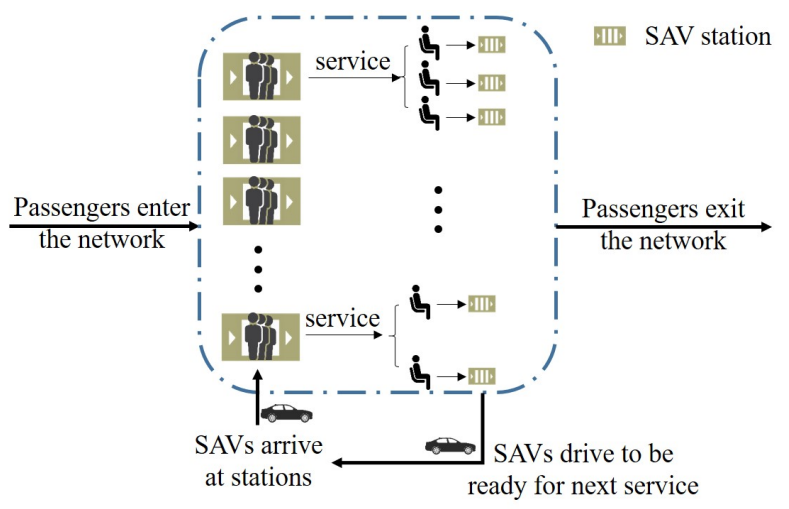

Fig. 2: Shared autonomous vehicles in a queueing system.

3) Agent-based modeling: To address the questions on the impact of SAVs on transportation mobility and investigate performance of the SAV system under various scenarios, several research efforts have also focused on developing agentbased models to evaluate the transportation network with presence of SAVs [61], [75], [76], [83]. With the advantage of modeling each individual passenger/vehicle as an agent following simple rules, complex behavior [84], [85] at a macroscopic level emerges, which provides an approximation of travel behavior in the transportation systems [75]. Marczuk et al. [86] and Azevedo et al. [87] proposed an extension to the agent-based demand and supply model (SimMobility) for the design and evaluation of the SAV system in a multi-level simulation framework, and explored the effects of fleet size and station location for both station-based and free-floating SAV systems. Boesch and Ciari [75] discussed the advantages of MATSim (an activity-based agent-based simulation model) with the presence of SAVs and its potential applications on investigating related problems, such as the potential of SAVs complementing or competing with other transportation modes, appropriate fleet size in different transportation systems, and the demand distribution with respect to the response of different fleet sizes.

Focusing on the potential impact of a SAV system on urban parking demand, Zhang et al. [77], [78] investigated different system operation strategies under low penetration of SAVs with an agent-based simulation model. Ridesharing and traveler's acceptance of sharing rides were also explored in the paper. The results showed a significant parking demand reduction with the SAV system - enabling ridesharing and adding vehicle cruising options would further reduce parking demand. Kondor et al. [88] developed an agent-based simulation model to estimate parking demand savings with shared vehicles and SAVs for home-work commuting. Other conclusions drawn from this study include that up to $50 \%$ reduction in parking needs could be achieved at the expense of less than 2\% increase in VMT. Jager et al. [83] developed an agent-based framework for a shared autonomous electric vehicle (SAEV) system that reflect the system behavior on an operational level. Although the system has a central dispatcher, the vehicles compete for customers and make their own decisions for routing and charging. Simulation results confirmed the feasibility of operating a SAV fleet with both high service levels and vehicle utilization. However, environmental benefits can only be expected when using renewable energy sources and enabling ride sharing features.

\section{SHARED AUTONOMOUS VEHICLE SYSTEM DESIGN VARIABLES}

Similar to conventional carsharing service, not only the operations of a SAV is significantly affected by the assignment and rebalancing strategies over a fleet of SAVs, mobility and environment, but also the urban landscape can be considerably impacted by the implementation strategies of a SAV system. Naturally, the problems of fleet sizing, vehicle-trip assignment, and rebalancing in a network of SAVs are the major subjects in enhancing our understanding of a SAV system, with the options of ridesharing and usage of electric vehicles that have attracted considerable attention recently. The majority of the literature to date has concentrated on how the SAV system tackles one or more of the aforementioned problems, and has aimed at enhancing our understanding about the performance and potential benefits of the network with a fleet of SAVs. In the following subsections, we provide a summary 
of SAV system modeling and discuss key topics that have been investigated in previous studies regarding the SAV system.

\section{A. Fleet Sizing of a Shared Autonomous Vehicles System}

Fleet size is the major determinant of the operating cost of the SAV system. General considerations in determining the fleet size include system access, directness, sharing, and passenger waiting time [89], [90]. In what follows, we summarize different approaches in addressing fleet sizing problems in a SAV system.

Fagnant et al. [91] simulated a SAV system in Austin area with a grid-based network model following a similar modeling framework presented in [43]. In this work, a fleet of SAVs is generated in the network to ensure that passenger waiting times are within predefined bounds. A heuristic strategy is implemented to relocate vehicles such that the stock of SAVs among cells is balanced. A replacement rate of $1 \mathrm{SAV}$ per 9.3 conventional vehicles was identified as appropriate for the area considered in the paper. The authors concluded that even with an excess VMT, emissions and environmental outcomes for the SAVs are still advantageous compared to those for the average US vehicle fleet. In the modeling framework for the SAV system developed by Winter et al. [89], the minimum fleet size and the optimal fleet size that yield the minimum system costs are determined through an iterative procedure, where the core is a simulation tool that is applied for assigning vehicles to passenger requests. Several scenarios are conducted to analyze the influence of different design parameters (e.g., vehicle capacity, operational parameters, demand level) on system performance.

Vazifeh et al. [92] investigated the minimum fleet size problem of a SAV system with a network-based model. Trips based on known demand and link travel times were taken as input to construct the vehicle shareability network under the constraint of maximum trip connection time. With fully knowledge of daily trip demand, the authors found that $40 \%$ taxis in New York City can be reduced without incurring delay to passengers, under the constraint of 15-minute maximum trip connection time. Relaxing the assumption of complete demand information, the authors concluded that if trip requests were collected at 1-minute interval, the system could be operated with a $30 \%$ fleet reduction at a relative high level of service (i.e., above $90 \%$ served trips within a 6-min delay).

Spieser et al. [73] addressed two major fleet sizing problems: (1) the minimum number of vehicles needed to stabilize the workload of a SAV system and (2) the number of vehicles needed to ensure a desired level of service provided to the customers. In their paper, the SAV system is modeled as a queueing network where each region is mapped into singleserver node, and each route between each pair of regions is mapped into infinite-server nodes. The vehicle rebalancing process is modeled as an arrival process of "virtual passengers." Conducting a case study in Singapore, the paper showed that a SAV can meet the personal mobility needs of the entire population with a fleet size about one third of the total number of passenger vehicles currently in operation.

Masoud and Jayakrishnan [93] discussed a different implementation strategy of the SAV system, with households form- ing clusters (i.e., neighborhoods). Each neighborhood share the ownership and ridership of a set of autonomous vehicles that serve as rental cars during their idling times. The authors focused on the optimization of the fleet size in a neighborhood and the number of rental requests for the vehicles during their idling times. Two optimization models were developed. The first model addressed the neighborhood clusters and aimed at minimizing the total number of the vehicles by considering essential trips to be satisfied for all the households in a neighborhood. The second model optimized the total number of rental requests so as to maximize extra income from idling vehicles, considering time window constraints of the owners' essential trips.

Most of previous work has emphasized on searching for the minimum fleet size of SAVs that could provide service on the existing demand at a desired level, when replacing the existing conventional vehicle service by SAVs. We have noticed promising results from multiple papers indicating that a high replacement rate of conventional vehicles is feasible to satisfy the same level of demand. However, there is still some work missing to assess holistically the impact of urban mobility due to potentially changing travel behavior and demand as a result of the introduction of $\mathrm{AV}$ in the mixed traffic environment.

\section{B. Vehicle Assignment in a Shared Autonomous Vehicle System}

Although there is a rich body in the literature in dynamic assignment problems with various applications on taxi, paratransit, trucking services, etc, that require real-time vehicle assignment to dynamic service requests (e.g., see [94]-[97] for more details), most papers reported in the literature to date have focused on investigating SAV system performance with simplified vehicle assignment strategies (usually rule-based). In what follows, we present a general formulation of the vehicle assignment problem in a SAV system. Let $i \in \mathcal{M}$ be a trip request, $j \in \mathcal{N}$ be the index of a vehicle, and $x_{i j}$ equal to 1 if and only if trip $i$ is assigned to vehicle $j$, where $\mathcal{M} \subset \mathbb{N}$ is the set of trip requests and $\mathcal{N} \subset \mathbb{N}$ is the set of vehicles. The general vehicle-traveler, or vehicle-trip, assignment problem to minimize the trip assignment cost, $J_{a}$, [38], [98]:

$$
\min J_{a}=\sum_{i} \sum_{j} c_{i j} x_{i j},
$$

subject to

$$
\begin{gathered}
\sum_{j} x_{i j}=1, i \in \mathcal{M}, \\
x_{i j} \in\{0,1\}, \forall i \in \mathcal{M}, j \in \mathcal{N},
\end{gathered}
$$

where $c_{i j}$ is the cost of assigning trip request $i$ to vehicle $j$, which could be represented by trip travel distance, travel time, or monetary cost. The trip assignment cost in (1) is evaluated at every trip assignment time step with dynamic service requests. The constraint 2 ensures that each traveler is assigned to only one vehicle.

When assigning travelers to the nearest idling AVs, several research efforts have considered a first-come-first-served strategy, which is a heuristic approach to minimize passenger waiting time [44], [78], [90], [91], [99]. In a paper by Fagnant 
and Kockelman [46], the SAV service area is divided into small zones, where trips are randomly generated. Every five minutes, passengers will be randomly ordered and assigned to the nearest available SAV in the same zone, up to a maximum vehicle arrival time. If such assignment fails, those passengers will be held until next assignment. Hyland and Mahmassani [100] investigated the underlying stochastic vehicle assignment problem for the SAV system with no shared rides. With the assumption that the fleet operator has no information of the spatial-temporal demand distribution, the authors compared different SAV assignment policies as the solution approaches to the local optimization problem at each time step. Two of the applied strategies were first-come-first-served, and the other strategies minimized traveler waiting times (under different vehicle-traveler assignment constraints).

Hanna et al. [101] examined four different methods for assigning vehicles in a SAV system: (1) a decentralized greedy matching where users are assigned to their nearest vehicles in a random order, (2) a centralized greedy matching approach ensuring that each vehicle is matched with its closest user, (3) the Hungarian minimum cost matching algorithm that minimizes passenger waiting time and unoccupied distance traveled, and (4) a minimal makespan matching algorithm which minimizes the longest distance that any vehicle must travel to a passenger. The authors showed that compared to greedy approaches, the latter two methods improved system performance through reducing unoccupied travel distance, passenger waiting time, and waiting time variation.

\section{Vehicle Rebalancing of a Shared Autonomous Vehicle Sys- tem}

The SAV system shares similar characteristics with the carsharing system consisting of conventional vehicles [46]. In terms of unbalanced demand distribution, both systems face the same problem of vehicle rebalancing. Two major rebalancing strategies have been investigated in the literature of carsharing with conventional vehicles including (1) operatorbased vehicle relocation and (2) user-based vehicle relocation, which could potentially be adapted in addressing the same problem in the SAV system, see [63], [102]-[105]. However, the relocation of SAVs still have differences with that of conventional sharing vehicles, since SAVs are fully compliant and always cooperative [106]. Thus, due to the inherent capabilities of self-driving and self-rebalancing of a SAV system, research efforts have focused more on the problem with a centralized operator that has dispatching control over the entire SAV network, which may yield a system optimum solution for the entire system.

We provide a general formulation to illustrate the vehicle rebalancing problem for a SAV system. Let $r_{y}$ be the number of idling vehicles in zone/station $y \in \mathcal{Z}$ and $r_{y z}$ be the number of rebalancing vehicles from zone/station $y$ to zone/station $z \in$ $\mathcal{Z}$, where $\mathcal{Z} \subset \mathbb{N}$ is the total number of zones/stations in the network. Generally, the objective function $J_{r}$ is the total cost induced by vehicle rebalancing [71], [107], [108]:

$$
\min J_{r}=\sum_{y} \sum_{z} c_{y z} r_{y z}
$$

subject to

$$
\begin{gathered}
\sum_{z} r_{y z}=r_{y}, \forall y, z \in \mathcal{Z}, \\
r_{y z} \in \mathbb{N}, \forall y, z \in \mathcal{Z},
\end{gathered}
$$

where $c_{y z}$ is the cost of moving vehicles from zone/station $y$ to zone/station $z$, which could be represented by trip travel distance, travel time, or monetary cost. In a system with dynamic trip requests, (4) will be evaluated at every rebalancing time step and (5) defines the total rebalancing vehicles from zone/station $y$ should equal the number of idling vehicles in the zone.

Targeting at the problem of unbalancing demand and supply, Pavone et al. [109] addressed the vehicle relocation problem for a mobility-on-demand system, optimizing the rebalancing assignment that minimizes the number of vehicles to be moved. Using a fluid model of the system, the authors showed that the optimal rebalancing policy can be found as the solution to a linear program, under which every station reaches an equilibrium where there are excess vehicles and no waiting customers. Based on this study, Zhang and Pavone [72] presented a queueing-theoretical approach and provided the solution to an offline optimal rebalancing problem. Later, Wen et al. [107] extended the research by incorporating doorto-door service and ridesharing option in a free-floating SAV system. From the fleet operator's perspective, Spieser et al. [108] investigated the vehicle rebalancing problem in a SAV system by quantifying the operation cost as a function of fleet size, demand loss and utilization rate, and analyzed the impact of fleet size on demand loss, vehicle utilization rate, and vehicle rebalancing miles traveled. Hörl et al. [110] evaluated performance of four heuristic and optimal rebalancing policies for a SAV system in an agent-based simulation environment, and suggested that the utilization of intelligent demand forecasts and rebalancing algorithms would be crucial for a SAV system to be competitive with private vehicles.

Through simulation based evaluation, recent work focused on the impact of vehicle rebalancing strategies in a SAV system. Zhu and Kornhauser [111] investigated the rebalancing strategies for the SAV system in New Jersey and their effects on the fleet size and level of service provided in scenarios where all non-walking travel demand is served by SAVs. Shared trips are served by vehicles of different capacities (i.e., $3,6,15$, and 50 passengers). Two rebalancing strategies are developed based on known demand. In the first approach, vehicles are moved at the end of the day to make sure that there are enough vehicles at each station that satisfy the demand at the beginning of the day. In the second approach, vehicles are relocated as needed to fill in any station without enough vehicles. The authors also evaluated the performance of the statewide SAV system with varying fleet sizes, in terms of passenger waiting time and rebalancing trip lengths. The results showed that one SAV could possibly replace more than six traditional vehicles while the demand could still be well served.

Fagnant and Kockelman [46] investigated the operation of SAVs through an agent-based model and focused on the implications of travel and environmental impacts of SAVs under a 
mixed traffic condition. Addressing the imbalanced demand patterns, the authors proposed several relocation strategies to balance vehicle supply and reduce future traveler wait times: (1) relocating vehicles based on expected demand and (2) relocating vehicles to balance stock based on predicted supply. Marczuk et al. [112] developed a simulation framework for rebalancing an one-way SAVs system in SimMobility environment. The proposed fleet management center is responsible for passenger-to-vehicle assignment, vehicle routing and re-balancing. Three vehicle relocation strategies were proposed for the system: (1) no rebalancing as the baseline scenario, (2) offline rebalancing that minimizes the number of rebalancing trips, and (3) online rebalancing that minimizes the total time/effort spent for rebalancing per rebalancing interval. Winter et al. [113] analyzed the impacts of different relocation strategies of a SAV system in a simulated generic grid network. Five vehicle relocation strategies were tested, including remaining idle, random shuffling, returning to original location, moving based on demand anticipation, and moving to balance vehicle stock over the network. In the simulation framework, the fleet size of the SAV system is given as an input, and vehicles are dispatched through a rule-based strategy. Performance measures such as average passenger utility, average waiting time, and the ratio of vehicle driving time were examined. The simulation showed that remaining idle strategy would be the most efficient in terms of passenger waiting time, yet the worst performer considering link occupancy and parking turnover rates. In contrast, strategies aimed at distributing vehicles yielded higher parking turnover rates but showed lower service efficiency. In light of these results, the authors extended the study by imposing the constraints of limited parking facilities in the evaluation of the above five heuristic relocation strategies for idle SAVs, and examined the potential impact of SAVs on urban traffic in terms of congestion, parking consumption and mode shift [114].

As discussed in the above papers, e.g., [111] $-[114]$, depending on the objectives and targeting performance measures, the rebalancing strategy to be applied in a SAV system may be different. The operation of a fleet of SAV is considerably affected by the applied relocation strategy or a combination of strategies, considering the inter-dependencies among parking demand, traffic condition, and user mode choice. Although current research efforts emphasize rebalancing strategies in an isolated SAV system, the externalities should be analyzed in more depth to enhance the understanding of traffic dynamics with the implementation of SAV service.

\section{The Usage of Electric Vehicles in a Shared Autonomous Vehicle System}

A significant amount of work has focused on the use of electric vehicles in a SAV system to achieve larger energy and emission savings for a greener transportation system [61], [76], [90]. Considering the range of electric vehicles, there is a number of constraints in a SAEV system. For instance, a vehicle may need to visit a charging station after dropping off passengers. There may be instances that vehicles have to turn down trip requests and drive to charging stations instead, resulting in different vehicle-trip assignment strategies [115][117].

Based on the work in [38], Zhang et al. [106] presented a model predictive control (MPC) approach to optimize vehicle scheduling and routing in a SAEV system, considering vehicle charging constraints. Compared to other control algorithms of a SAV system (i.e., nearest-neighbor dispatch, collaborative dispatch, Markov redistribution, real-time rebalancing), the authors concluded with a case study in New York City that the MPC algorithms outperformed the other strategies in terms of average customer waiting times.

Chen et al. [61], [76] addressed the operations of a SAEVs with an agent-based model based on the work reported in [46] and [91]. The emphasis of this research is the performance analysis of a fleet of SAEVs under various vehicle range and charging infrastructure scenarios. The authors also explored the pricing schemes of a SAEV system when competing against other modes (i.e., private human-driven vehicles and city bus service), and found that with higher SAEV penetration rate, the private vehicle replacement rate by the SAEVs increases, leading to improved system performance. Similarly, the study by Bauer et al. [118] predicted battery range and charging infrastructure requirements of a fleet of SAEVs operating on Manhattan island with an agent-based model. The authors also conducted sensitivity analysis of the cost and the environmental impact of providing SAEV service with a wide range of changes in cost components (e.g., battery type, vehicle type, etc.). The study indicated that instead of battery range, the major challenge to introducing SAEVs may be building sufficient charging infrastructure.

Kang et al. [115] developed a framework for a SAEV system that consists of demand forecasting, fleet assignment, electric vehicle designing, and charging station locating modules. The fleet assignment module determines the optimal vehicle assignment and charging schedules, and the charging station locating module decides the optimal charging station locations. The system-level objective is to maximize service profit for the operator, through optimizing decision variables including fleet size, number of charging stations, electric powertrain design, membership fee, and vehicle rental fee. The locations of charging stations are selected with a p-median model from a pool of predetermined candidates. A comparison between a SAV system and a SAEV system was conducted in terms of cost and benefit under different scenarios (e.g., varying gas prices and charging station installation costs), showing that a SAEV system would be more profitable for most of the scenarios. Although both systems are marketable, the optimized SAEVs required longer waiting times than optimized SAVs due to the constraints of vehicle range and charging issues.

Iacobucci et al. [119] developed a simulation model to evaluate a SAEV system interacting with passengers and charging at designated stations based on a heuristic charging strategy. The potential utilization of the SAEV system as an operating reserve provider and its performance in response to grid operator requests were evaluated. The authors concluded that the proposed system could reduce the required fleet size as compared to private vehicles while providing a comparable level of transportation service with low break-even prices. 
Later, based on the work presented in [106], the authors developed a framework for the optimization of charging scheduling and vehicle routing and relocation for a fleet of SAEVs [120]. The proposed framework consists of two layers of optimization model: over longer time scales, the charging scheduling optimization minimizes waiting times and electricity costs, while over shorter time scales, vehicle routing and relocation are optimized under charging constraints. The authors reported that a substantial reduction in charging costs was yielded from the proposed framework without significantly affecting passenger waiting times, as well as the potential of SAEVs to offer energy storage to the grid and avoid grid congestion.

In summary, the introduction of electric vehicles in the SAV system offers a large potential to further enhance environmental benefits. However, constraints such as vehicle range and charging facility locations add more dynamics into the system, and multiple studies suggested that the infrastructure and charging scheduling are the key influencing factors of system performance of a fleet of SAEVs. Considerably work has focused on the performance analysis of SAEV system as compared to the SAV system, through evaluating the impact of vehicle range, charging infrastructure, as well as electricity costs [61], [76], [118]. Considering charging constraints, several research efforts have also emphasized on re-examining vehicle routing and relocation strategies as well as optimizing charging locations [106], [115], [121]. Recently, the option of vehicle-to-grid as well as the integrated planning of power grid and shared mobility service has also attracted considerable attention [119], [120], to improve the perception of SAEVs and ensure sustainable commutes within the notion of smart cities [122].

\section{E. The Option of ridesharing in a Shared Autonomous Vehicle System}

The problems of ridesharing and carsharing are usually decoupled in the existing literature [123]. Recently research efforts started exploring the option of ridesharing in a SAV system, e.g., [124]-[127]. By allowing ridesharing, the fleet size may be further reduced to provide a desired level of service to the passengers, although the total VMT probably might increase [128], [129]. There are generally two types of ridesharing as illustrated in Fig. 3 (a) trip combining neighboring origins and destinations (Fig. 3a and (b) trip chaining based on trip temporal and spatial characteristics (Fig. 3b). We consider here ridesharing as the option of serving multiple passengers in a single vehicle trip, or trip chain, in the SAV system, and emphasize the impact of opening up ridesharing options in the SAV service, without detailing the operation modes and strategies for ridesharing. Considering different system objectives (e.g., minimizing total VMT, minimizing total travel time, or maximizing served trips) and various system constraints (e.g., time window and seat constraints), there has been work on the SAV system with the option of ridesharing and the evaluation of different ridesharing strategies against network performance.

Levin et al. [124] analyzed the possibility of ridesharing in a SAV system where passengers could select the first arrived

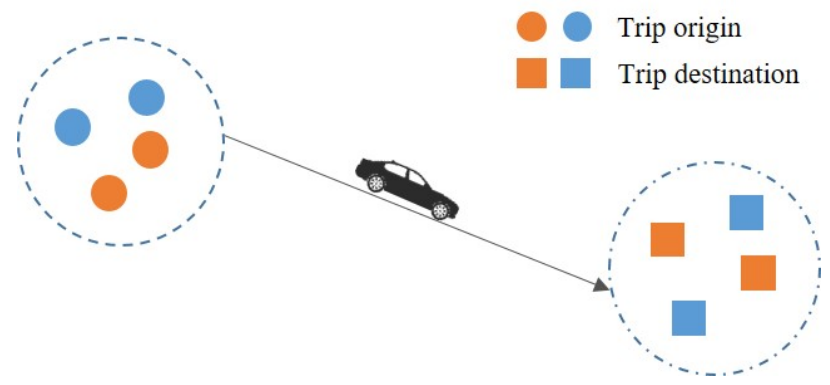

(a)

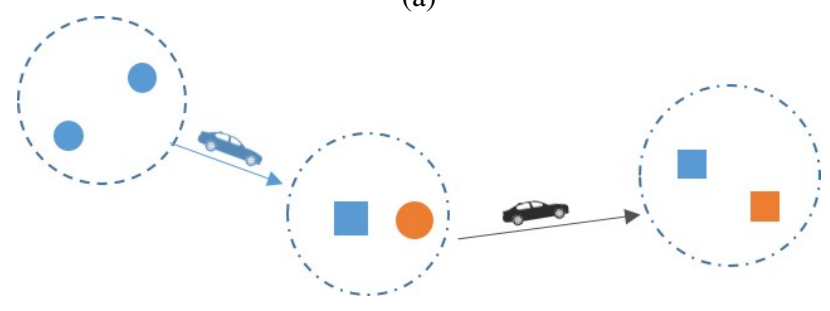

(b)

Fig. 3: ridesharing in the shared autonomous vehicle system: a) trip combination; b) trip chaining.

vehicle regardless of occupancy. The authors found that SAVs with the choice of ridesharing may cause more congestion due to additional miles traveled for detouring. Zhang et al. [78], [142] applied an agent-based model to evaluate the performance and potential benefits of a SAV system with dynamic ridesharing. In a grid-based simulation network, a centralized operator monitors real-time trip requests and SAV status as well as manages trip assignment for the SAV system, where ridesharing option is evaluated against passenger's willingness and travel cost. Their work suggested that dynamic ridesharing in a SAV system could potentially lead to reduced vehicle ownership, parking demand, and emissions.

Hyland and Mahmassani [125] compared the performance of a SAV system with and without ridesharing option in terms of the ability to handle demand surges. In this paper, the mathematical formulations of the vehicle assignment with/without ridesharing were presented and the solutions were derived with a rolling-horizon approach. The simulation results indicated that the SAV with ridesharing service improved system performance in response to demand surges.

Based on the vehicle rebalancing strategies tested in [46], Fagnant and Kockelman [126] considered the option of dynamic ridesharing in a simulated SAV system. With the case study of a 24-mile by 12-mile region in Austin, the authors concluded that dynamic ridesharing in a SAV system was able to limit excess VMT from the SAV system, reduce passenger waiting times (under the constraint that ridesharing should not increase travel time of current passengers by more than $40 \%$ ), and yield an enhanced level of service.

Farhan and Chen [141] discussed the impacts of ridesharing on the operational efficiency of SAEVs with a discrete-time simulation model. Both the fleet size and number of charging stations are determined during simulation. In their research, the travelers are grouped into clusters based on spatial criteria, and the ride-share matching problem is formulated as a vehicle 
TABLE I: Approaches in Shared Autonomous Vehicle System Modeling.

\begin{tabular}{|c|c|c|}
\hline Approach & Topic & Reference \\
\hline Optimization & $\begin{array}{l}\text { Fleet sizing } \\
\text { Vehicle routing / trip assignment } \\
\text { Vehicle rebalancing / relocation } \\
\text { Other considerations }\end{array}$ & 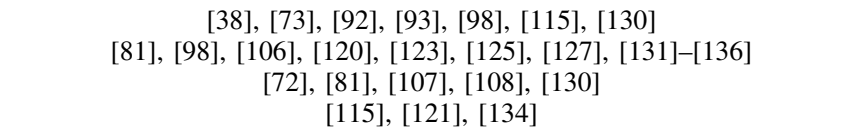 \\
\hline Simulation Evaluation & $\begin{array}{l}\text { Fleet sizing } \\
\text { Vehicle routing / trip assignment } \\
\text { Vehicle rebalancing / relocation } \\
\text { Ridesharing } \\
\text { Pricing scheme } \\
\text { Transit integration / mode choice } \\
\text { Electric vehicles }\end{array}$ & 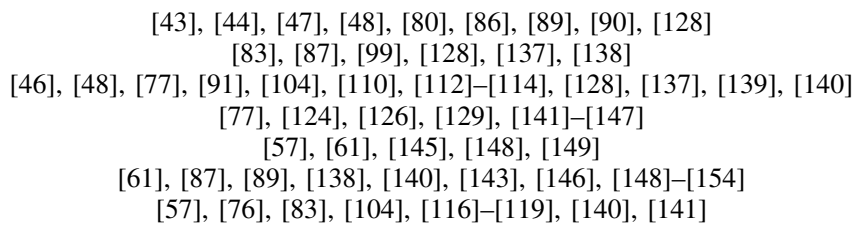 \\
\hline
\end{tabular}

routing problem minimizing system-wide vehicle miles traveled under time window constraint. The results indicated that allowing a second passenger in ridesharing yielded marginal benefit of fleet size and charging station reduction. Although more passengers in shared trips reduced the required fleet size and number of charge stations, passenger waiting times increased due to ridesharing (i.e., reduced level of service).

\section{Shared Autonomous Vehicle System Operation}

Although the majority of the literature has been focused on examining the feasibility and performance of the SAV service as an isolated system, there is an increasing interest towards the investigation of more realistic operational scenarios related to the SAVs. Recent research efforts have also focused on answering questions such as: "How will the SAV system perform in a mixed traffic environment?" "What will be the mobility impact of integrating the SAVs with other modes of transport?" In this section, we focus on different operational aspects of a SAV system, and summarize the studies that consider realistic and mixed traffic conditions.

\section{A. Operation in a Realistic Traffic Environment}

The majority of the aforementioned work has addressed the SAV system with full SAV penetration or without considering background traffic. Only a few papers have focused on the impact of congestion of SAVs, e.g., [81], [124], [132], [155], [156]. For example, to investigate the impact of SAVs on mobility, Levin et al. [124] presented a general event-based framework for simulating the operations of a SAV system with existing traffic models. Considering $100 \%$ penetration of SAVs, the authors found that under certain scenarios (e.g., with the option of dynamic ridesharing), a smaller fleet of SAVs performed better than a larger fleet due to lower congestion in the network. Maciejewski and Bischoff [156] evaluated the impact of a city-wide introduction of SAVs on traffic congestion through an agent-based simulation model, focusing on the analysis of traffic congestion under different SAV penetration rates. Under the assumption of increased road capacity due to AV operations, their work showed that despite increased traffic volume, a fleet of SAV could have a positive effect on traffic at a penetration rate as low as $20 \%$.

Levin [131] developed a linear programming formulation for vehicle routing problem in the SAV system, where traffic flow was modeled through the link transmission model. The results showed that asymmetric demand (e.g., demand during peak periods) could lead to significantly rebalancing trips and greater congestion than uniformly distributed demand pattern. Since more vehicles might cause additional congestion on roadway network, it is important for the SAV system to plan for different traffic patterns. Liang et al. [132] proposed an integer programming model to define the routing of the SAVs based on profit maximization function, where travel times on the links varied with the flow of SAVs (without any background traffic). Later in [133], the authors applied the algorithm for trip assignment and dynamic routing in the city of Delft, the Netherlands with a rolling horizon scheme. Assuming that the operator of a SAV fleet has the choice of accepting or rejecting trip requests according to a profit maximization function, the analysis showed that taking into account the impact of dynamic travel time led to different results of satisfied trips and VMT, and ultimately affected overall operator profit and network congestion level.

Rossi et al. [81] studied the routing and rebalancing problem of SAVs in congested transportation networks, where a SAV system is modeled in a network flow framework such that vehicles are represented as flows in a road network. The objective of the routing problem is to minimize the weighted sum of passenger trip travel times and vehicle rebalancing travel times considering network capacity. The objective of the rebalancing problem is to optimize rebalancing paths such that traffic congestion is minimized. Through numerical studies on real-world traffic data, the authors showed that the proposed real-time routing and rebalancing algorithm yielded lower customer waiting time by avoiding excess congestion on the road, compared to point-to-point rebalancing algorithms where no underlying road network is assumed.

Through an agent-based model, Fagnant and Kockelman [46] investigated the operation of SAVs and focused on the implications of travel and environmental impacts of SAVs under a mixed traffic condition. Instead of $100 \%$ penetration of SAVs, the authors considered the transportation system with a small market share of SAVs (i.e., around 3.5\%). The simulation results under different scenarios (e.g., varying trip generation rates, network congestion levels, SAV fleet size, etc) indicated that each SAV can substitute around eleven conventional vehicles at the cost of $10 \%$ more VMT, and the overall emissions savings are expected to be sizable for most emission species. 


\section{B. Operating in a Multi-Modal Environment}

Based on the discussion in the previous sections, it seems clear that SAVs, compared to personal owned human-driven vehicles, have significant advantages for individuals as well as for the transportation system in terms of mobility, safety, and energy savings (especially with SAEVs), e.g., [46]-[48], [76], [83], [86]. A combination of SAVs with other transportation modes such as public transportation, however, might impose different conclusions [9], [10], [12], [18]. Although SAVs could be utilized in the way to facilitate the first and last mile transport [157] and promote the use of public transportation system (e.g., [154], [158]), SAVs may also divert passengers away from transit systems due to their capability of providing door-to-door services (e.g., [154], [159]).

1) Shared autonomous vehicles as a complement of public transit: Early research efforts have explored the performance of integrating the SAV system with transit systems. For example, based on the same network in New Jersey as in [43], Zachariah et al. [143] simulated a system of SAVs where the train network is preserved and treated as an integral part of the system. Using SAVs as a complementary service of a train system, Liang et al. [134] presented an optimization model to define the service area of a SAV system for first/last mile transport that maximizes the profit of the SAV operator. Later in [135], the authors designed a SAV system providing shuttle service between a major train station and city area, considering the competition between SAVs or other modes (e.g., biking or walking), as well as the impact of traffic congestion on mode split. With the objective of minimizing total travel time, the authors developed an optimization model to decide the best fleet size and price rate for the SAV system.

Shen et al. [146], [151] explored the feasibility of integrating SAVs in the public transportation system to improve the first/last mile connectivity. With a simplified simulation model without considering traffic congestion where the demand for the SAV system was assumed to be $10 \%$ of the original bus demand, the study showed that by enabling ridesharing, the integrated service was able to reduce average passenger travel time and ease traffic through less occupancy of road resources. Scheltes and de Almeida Correia [140] studied the SAEV system providing last-mile service for a train line. In the simulation model, vehicle assignment in response to traveler request followed a first-come-first-served model. The scenarios of short-term pre-booking, vehicle relocating, and opportunity charging were also explored. The results showed that compared to bicycle and walking as last mile transportation modes, the SAEV system was able to reduce average passenger travel time and waiting time, especially when pre-booking option was enabled.

Wen et al. [153] proposed a systematic approach to design and simulate an integrated system of SAVs and public transit. The authors emphasized that the SAV operation is designed to be transit-oriented with the purpose of supporting existing public transit service. In an agent-based simulation platform, the interaction between service operator and travelers is modeled with a set of system dynamics equations, such that the decisions of both parties could be captured in the system. The authors suggested that encouraging ridesharing, allowing inadvance requests, and combining fare with transit would be useful to enable service integration and promote sustainable travel. Pinto et al. [138] proposed a simulation framework integrating a travel mode choice model and a dynamic transit assignment model to assess the impacts of a suburban firstmile SAV system on transit demand. Similarly, Martinez and Viegas [150] presented an agent-based model to evaluate the impact of the SAVs in the city of Lisbon, Portugal. In their simulation model, current travel demand is served by two types of AVs that compete with each other, i.e., a SAV providing door-to-door service with the choice of ridesharing and an autonomous minibus that replaces current bus service without any transfers for users. The simulation results revealed positive mobility impact of SAVs especially when introducing the autonomous minibus into the network.

2) Shared autonomous vehicles as a competitor of public transit: Liu et al. [149] simulated transportation patterns in Austin network with a system of SAV from a mode-choice perspective. A user-equilibrium based dynamic traffic assignment model was applied in simulation environment. The study focused on travelers' mode choices with the presence of SAVs. In a mixed traffic environment, where private human-driven vehicles, public transit, and SAVs coexist, the study analyzed the impacts of the SAV system on energy consumption and emissions under different SAV penetration rates and SAV rental fees. Based on the sensitivity analysis of rental fees, the authors found that if the SAV fare rate is low enough, SAV users might travel more than private vehicle users. Therefore, although the use of AVs is expected to result in energy savings and emission reduction, the extra VMT by SAVs could compromise such environmental benefits. The mode choice results indicated that, for travelers who do not own a private vehicle, SAVs are preferable for short-distance trips compared to public transit. However, demand shifting from public transit would be a concern once the SAVs become available in the study area. Hörl [148] conducted a similar study and investigated the SAV service in a multi-modal traffic simulation environment. The simulation results in the test scenario raised the following two concerns: (1) the introduction of SAVs led to increased VMT and, moreover, (2) SAVs attracted public transportation users rather than private car owners.

Snelder et al. [152] developed a simulation framework to assess both direct and indirect impacts of AVs and SAVs in a mixed traffic environment. To capture demand elasticities, the network fundamental diagram was combined with mode choice models. Furthermore, the spatial impact was also modeled as an exogenous input to the framework via a percentage of relocated inhabitants per lane use type. The simulation results showed that a shift to SAVs could be expected. However, the improved accessibility for many residents could result in a significant increase in vehicle trips (and also in VMT), which might impose negative effects on traffic condition. Similar conclusions were drawn from the study on the effects of full automation with the possibility of trip chaining of household trips, yet in a scenario where most vehicles are still privately owned [136]. 
In summary, findings of multiple studies indicate that although the introduction of SAVs in the transportation system might improve mobility and safety, it could result in enormous changes of travel behavior, mode choice, car ownership, and possibly transportation infrastructure and urban form. A holistic assess of the impact of the SAV systems on urban mobility and related social implications might be challenging at the moment as SAVs are still evolving. However, SAV service could possibly have negative impact on traffic congestion and be strongly competitive with public transit without appropriate incentive mechanisms.

\section{Outlook And Future Directions}

\section{A. Concluding Remarks}

In this paper, we summarized current research efforts in SAV systems that have been reported in the literature to date. Although the SAV system have many aspects in common with the conventional carsharing system, the inherent characteristics of self-driving and self-rebalancing with SAVs further enhance free-floating carsharing service and increase the stochasticity of the system internally. Externally, the introduction of AVs in the transportation network could change fundamentally traffic patterns in the future. The complexity of traffic and urban dynamics, thus, places considerable uncertainty in terms of both short-term and long-term impacts of the system [160].

The majority of research efforts has considered a system either of full SAV penetration rate or without any traffic, and compared its performance with the conventional mobility systems (in terms of fleet size requirement, energy implications, VMT, passenger travel times, etc). Among these research efforts, agent-based modeling is one of the major approaches to evaluate network performance of a SAV system and assess potential impacts of the system. Several research efforts have focused on developing optimization models to address the following questions: (1) "what is the minimum fleet size to provide a desired level of service?" (2) "What is the optimal vehicle assignment strategy to minimum passenger travel time?" (3) "What is the optimal vehicle relocation strategy to minimize the number of rebalancing trips without inducing waiting delay?" In general, the SAV system could benefit from the cooperative characteristics of the fleet the connectivity and automation embedded in the system open up the opportunities for a central controller to apply optimal operation strategies to achieve global optimum against different network design objectives.

Although previous research has aimed at enhancing our understanding of the SAV systems, there are still open issues to be addressed. For example, most papers consider the SAV system with fixed stations whereas free floating SAV systems have not been thoroughly investigated. Within a SAV system, the optimal fleet sizing problem to maintain a minimum required level of service or to ensure a desired level of service is still under-explored. The considerations of different vehicle assignment and relocation strategies, or the option of ridesharing further increase the complexity of the problem. So far most papers have applied heuristics for the implementation of SAVs to solve these problems and focused more on assessing potential benefits of a SAV system.

\section{B. Future Research}

There are several directions for future research considering the gaps in the work reported in the literature to date. Although previous work has addressed the replacement ratio of SAVs to conventional private vehicles, the majority of the results are derived with existing demand patterns in an isolated system. The problem of modeling the SAV system with presence of other transportation modes, as either a complement or competing mode, needs further investigation. Especially, the following questions still remain unanswered: (1) "What is the network performance of a SAV system in a realistic transportation network?" (2) "How much improvement in the level of service in a transportation network can be achieved with an integrated SAV system?" To address these challenges, it is necessary to study the operational strategies (e.g., optimal fleet size/vehicle assignment/relocation strategy, etc) which would yield the minimum and/or desired level of service of the transportation network. Furthermore, in an environment where massive amount of data could be collected from vehicles and infrastructure, what we used to model as uncertainty become an additional input. With the advent of information and communication technologies, better utilizing available information for optimal operational strategies requires novel solutions to reduce dimensions and to overcome issues associated with data in high-dimensional spaces.

With all possible mobility service options enabled by CAVs, one particular question that still remains unanswered is "how demand pattern or travel behavior will eventually change?" With the shared mobility choices (and enhanced convenience with SAVs), there is already an evidence of an increase of induced demand (e.g., more night travels, or trips shifted from transit demand). However, little research has been conducted on investigating the impact of the emerging SAV system on the vulnerable population, while a systematic framework of providing accessibility to a variety of social groups is still missing. Meanwhile, the nature of self-driving and selfrebalancing of a SAV system also implies potential changes on land use. For example, the implications of a SAV system on urban parking spaces is still under-explored. Thus, the longterm impact of shared mobility system on urban transportation systems is still an open question.

\section{REFERENCES}

[1] A. A. Malikopoulos, "A duality framework for stochastic optimal control of complex systems," IEEE Transactions on Automatic Control, vol. 61, no. 10, pp. 2756-2765, 2016.

[2] A. Malikopoulos, "Convergence properties of a computational learning model for unknown markov chains," J. Dyn. Sys., Meas., Control., vol. 131, no. 3, pp. 041 011-7, 2009.

[3] A. Malikopoulos, P. Papalambros, and D. Assanis, "A real-time computational learning model for sequential decision-making problems under uncertainty," J. Dyn. Sys., Meas., Control, vol. 131, no. 4, pp. 041010 8, 2009.

[4] S. Shaheen and N. Chan, "Mobility and the sharing economy: Potential to facilitate the first-and last-mile public transit connections," Built Environment, vol. 42, no. 4, pp. 573-588, 2016.

[5] S. Shaheen, A. Cohen, and I. Zohdy, "Shared Mobility: Current Practices and Guiding Principles," Federal Highway Administration, Tech. Rep. Washington D.C., 2016.

[6] SAE, "Taxonomy and Definitions for Terms Related to Shared Mobility and Enabling Technologies," SAE International, Tech. Rep., 2018. 
[7] A. Cohen and S. Shaheen, Planning for shared mobility. American Planning Association, 2018.

[8] C. Standing, S. Standing, and S. Biermann, "The implications of the sharing economy for transport," Transport Reviews, vol. 39, no. 2, pp. 226-242, 2019.

[9] S. Feigon and C. Murphy, Shared mobility and the transformation of public transit. American Public Transportation Association, 2016, no. Project J-11, Task 21.

[10] S. Shaheen and A. Cohen, "Is it time for a public transit renaissance? Navigating travel behavior, technology, and business model shifts in a brave new world," Journal of Public Transportation, vol. 21, no. 1, p. 8,2018 .

[11] G. G. D. Santos, "One-Way Carsharing Systems: Real-Time Optimization of Staff Movements and Operations," Ph.D. dissertation, Universidade de Lisboa, 2016.

[12] E. Martin and S. Shaheen, "Impacts of car2go on vehicle ownership, modal shift, vehicle miles traveled, and greenhouse gas emissions: An analysis of five North American cities," Transportation Sustainability Research Center, UC Berkeley, 2016.

[13] _ " "The impact of carsharing on public transit and non-motorized travel: An exploration of North American carsharing survey data," Energies, vol. 4, no. 11, pp. 2094-2114, 2011.

[14] E. Martin and S. A. Shaheen, "Greenhouse gas emissions impacts of carsharing in North America," Transactions on Intelligent Transportation Systems, vol. 12, no. 4, pp. 1-114, 2011

[15] J. Firnkorn and M. Müller, "Free-floating electric carsharing-fleets in smart cities: The dawning of a post-private car era in urban environments?" Environmental Science \& Policy, vol. 45, pp. 30-40, 2015.

[16] T. D. Chen and K. M. Kockelman, "Carsharing's life-cycle impacts on energy use and greenhouse gas emissions," Transportation Research Part D: Transport and Environment, vol. 47, pp. 276-284, 2016.

[17] M. Barth and S. Shaheen, "Shared-use vehicle systems: Framework for classifying carsharing, station cars, and combined approaches," Transportation Research Record: Journal of the Transportation Research Board, vol. 1791, pp. 105-112, 2002.

[18] J. Lazarus, S. Shaheen, S. E. Young, D. Fagnant, T. Voege, W. Baumgardner, J. Fishelson, and J. S. Lott, "Shared automated mobility and public transport," in Road Vehicle Automation 4. Springer, 2018, pp. $141-161$

[19] F. Duarte and C. Ratti, "The impact of autonomous vehicles on cities: A review," Journal of Urban Technology, vol. 25, no. 4, pp. 3-18, 2018

[20] P. Hancock, I. Nourbakhsh, and J. Stewart, "On the future of transportation in an era of automated and autonomous vehicles," Proceedings of the National Academy of Sciences, p. 201805770, 2019.

[21] S. Shaheen, A. Cohen, B. Yelchuru, S. Sarkhili, B. A. Hamilton et al., "Mobility on demand operational concept report," United States. Department of Transportation. Intelligent Transportation ..., Tech. Rep., 2017.

[22] P. Tientrakool, Y. C. Ho, and N. F. Maxemchuk, "Highway capacity benefits from using vehicle-to-vehicle communication and sensors for collision avoidance," in Vehicular Technology Conference (VTC Fall), 2011. IEEE, 2011, pp. 1-5.

[23] S. Shladover, D. Su, and X.-Y. Lu, "Impacts of cooperative adaptive cruise control on freeway traffic flow," Transportation Research Record: Journal of the Transportation Research Board, vol. 2324, no. 1, pp. 63-70, 2012.

[24] D. J. Fagnant and K. Kockelman, "Preparing a nation for autonomous vehicles: Opportunities, barriers and policy recommendations," Transportation Research Part A: Policy and Practice, vol. 77, pp. 167-181, 2015.

[25] R. Krueger, T. H. Rashidi, and J. M. Rose, "Preferences for shared autonomous vehicles," Transportation research part C: emerging technologies, vol. 69, pp. 343-355, 2016.

[26] T. Litman, "Autonomous Vehicle Implementation Predictions: Implications for Transport Planning," Victoria Transport Policy Institute, Tech. Rep., 2018

[27] A. A. Malikopoulos and L. Zhao, "A closed-form analytical solution for optimal coordination of connected and automated vehicles," in 2019 American Control Conference, 2019 (to appear).

[28] J. Rios-Torres and A. A. Malikopoulos, "A Survey on Coordination of Connected and Automated Vehicles at Intersections and Merging at Highway On-Ramps," IEEE Transactions on Intelligent Transportation Systems, vol. 18, no. 5, pp. 1066-1077, 2017.
[29] A. A. Malikopoulos, C. G. Cassandras, and Y. Zhang, "A decentralized energy-optimal control framework for connected automated vehicles at signal-free intersections," Automatica, vol. 93, pp. 244 - 256, 2018.

[30] L. Zhao, A. A. Malikopoulos, and J. Rios-Torres, "Optimal control of connected and automated vehicles at roundabouts: An investigation in a mixed-traffic environment," in 15th IFAC Symposium on Control in Transportation Systems, 2018, pp. 73-78.

[31] A. Stager, L. Bhan, A. A. Malikopoulos, and L. Zhao, "A scaled smart city for experimental validation of connected and automated vehicles," in 15th IFAC Symposium on Control in Transportation Systems, 2018, pp. $120-135$.

[32] A. A. Malikopoulos, S. Hong, B. Park, J. Lee, and S. Ryu, "Optimal control for speed harmonization of automated vehicles," IEEE Transactions on Intelligent Transportation Systems, 2019 (in press).

[33] J. Rios-Torres, A. A. Malikopoulos, and P. Pisu, "Online Optimal Control of Connected Vehicles for Efficient Traffic Flow at Merging Roads," in 2015 IEEE 18th International Conference on Intelligent Transportation Systems, 2015, pp. 2432-2437.

[34] A. A. Malikopoulos, P. Papalambros, and D. Assanis, "Optimal engine calibration for individual driving styles," in SAE Congress, 2008.

[35] A. A. Malikopoulos, P. Y. Papalambros, and D. N. Assanis, "Online identification and stochastic control for autonomous internal combustion engines," Journal of Dynamic Systems, Measurement, and Control, vol. 132, no. 2, pp. 024 504-024 504, 2010.

[36] A. A. Malikopoulos, Real-Time, Self-Learning Identification and Stochastic Optimal Control of Advanced Powertrain Systems. ProQuest, 2008 .

[37] S. Shaheen and M. Galczynski, "Autonomous carsharing/taxi pathways," in TRB Automated Vehicles Symposium, 2014.

[38] R. Zhang, K. Spieser, E. Frazzoli, and M. Pavone, "Models, algorithms, and evaluation for autonomous mobility-on-demand systems," in American Control Conference (ACC), 2015. IEEE, 2015, pp. 2573-2587.

[39] S. Rodoulis, "The impact of autonomous vehicles on cities," Journeys - Sharing Urban Transport Solutions, no. 12, pp. 12-20, 2014.

[40] W. Gruel and J. M. Stanford, "Assessing the long-term effects of autonomous vehicles: A speculative approach," Transportation Research Procedia, vol. 13, pp. 18-29, 2016.

[41] M. Ohnemus and A. Perl, "Shared autonomous vehicles: Catalyst of new mobility for the last mile?" Built Environment, vol. 42, no. 4, pp. 589-602, 2016.

[42] A. Stocker and S. Shaheen, "Shared automated vehicle (sav) pilots and automated vehicle policy in the us: Current and future developments," in Road Vehicle Automation 5. Springer, 2019, pp. 131-147.

[43] H. J. Ford, "Shared autonomous taxis: Implementing an efficient alternative to automobile dependency," B.S. Thesis, Department of Operations Research and Financial Engineering, Princeton University, 2012.

[44] L. D. Burns, W. C. Jordan, J. Analytics, and B. A. Scarborough, "Transforming Personal Mobility," The Earth Institute, Columbia University, Tech. Rep., 2012.

[45] Z. Chong, B. Qin, T. Bandyopadhyay, T. Wongpiromsarn, B. Rebsamen, P. Dai, E. Rankin, and M. H. Ang, "Autonomy for mobility on demand," in Intelligent Autonomous Systems 12. Springer, 2013, pp. 671-682.

[46] D. J. Fagnant and K. M. Kockelman, "The travel and environmental implications of shared autonomous vehicles, using agent-based model scenarios," Transportation Research Part C: Emerging Technologies, vol. 40, pp. 1-13, 2014

[47] J. Bischoff and M. Maciejewski, "Simulation of city-wide replacement of private cars with autonomous taxis in Berlin," Procedia Computer Science, vol. 83, pp. 237-244, 2016.

[48] H. Dia and F. Javanshour, "Autonomous shared mobility-on-demand: Melbourne pilot simulation study," Transportation Research Procedia, vol. 22, pp. 285-296, 2017.

[49] L. A. Merlin, "Comparing automated shared taxis and conventional bus transit for a small city," Journal of Public Transportation, vol. 20, no. 2, pp. 19-39, 2017

[50] P. M. Bösch, F. Becker, H. Becker, and K. W. Axhausen, "Cost-based analysis of autonomous mobility services," Transport Policy, vol. 64, pp. 76-91, 2017.

[51] A. Moorthy, R. De Kleine, G. Keoleian, J. Good, and G. Lewis, "Shared autonomous vehicles as a sustainable solution to the last mile problem: A case study of Ann Arbor-Detroit area," SAE International Journal of Passenger Cars - Electronic and Electrical Systems, vol. 10, no. 2, pp. 2017-01-1276, 2017.

[52] D. Metz, "Developing policy for urban autonomous vehicles: Impact on congestion," Urban Science, vol. 2, no. 2, p. 33, 2018. 
[53] N. Menon, N. Barbour, Y. Zhang, A. R. Pinjari, and F. Mannering, "Shared autonomous vehicles and their potential impacts on household vehicle ownership: An exploratory empirical assessment," International Journal of Sustainable Transportation, vol. 8318, pp. 1-12, 2018.

[54] D. Földes and C. Csiszár, "Framework for planning the mobility service based on autonomous vehicles," in 2018 Smart City Symposium Prague (SCSP). IEEE, 2018, pp. 1-6.

[55] L. T. Truong, C. De Gruyter, G. Currie, and A. Delbosc, "Estimating the trip generation impacts of autonomous vehicles on car travel in Victoria, Australia," Transportation, vol. 44, no. 6, pp. 1279-1292, 2017.

[56] I. P. Tussyadiah, F. J. Zach, and J. Wang, "Attitudes toward autonomous on demand mobility system: The case of self-driving taxi," in Information and Communication Technologies in Tourism 2017. Springer 2017, pp. 755-766.

[57] F. Dandl and K. Bogenberger, "Comparing future autonomous electric taxis with an existing free-floating carsharing system," IEEE Transactions on Intelligent Transportation Systems, pp. 1-11, 2018.

[58] A. Stocker and S. Shaheen, "Shared automated mobility: early exploration and potential impacts," in Road Vehicle Automation 4. Springer, 2018, pp. 125-139.

[59] _ - "Shared automated vehicles: Review of business models," in Roundtable on Cooperative Mobility Systems and Automated Driving, International Transport Forum Discussion Paper. International Transport Forum, 2017.

[60] C. Ross and S. Guhathakurta, "Autonomous vehicles and energy impacts: a scenario analysis," Energy Procedia, vol. 143, pp. 47-52, 2017

[61] T. D. Chen and K. M. Kockelman, "Management of a shared autonomous electric vehicle fleet," Transportation Research Record: Journal of the Transportation Research Board, vol. 2572, no. 2572, pp. 37-46, 2016.

[62] P. Davidson, A. Spinoulas et al., "Driving alone versus riding togetherhow shared autonomous vehicles can change the way we drive," Road \& Transport Research: A Journal of Australian and New Zealand Research and Practice, vol. 25, no. 3, p. 51, 2016.

[63] D. Jorge and G. Correia, "Carsharing systems demand estimation and defined operations: A literature review," European Journal of Transport and Infrastructure Research, vol. 13, no. 3, pp. 201-220, 2013.

[64] N. Agatz, A. Erera, M. Savelsbergh, and X. Wang, "Optimization for dynamic ride-sharing: A review," European Journal of Operational Research, vol. 223, no. 2, pp. 295-303, 2012.

[65] M. Furuhata, M. Dessouky, F. Ordóñez, M.-E. Brunet, X. Wang, and S. Koenig, "Ridesharing: The state-of-the-art and future directions," Transportation Research Part B: Methodological, vol. 57, pp. 28-46, 2013.

[66] G. Brandstätter, C. Gambella, M. Leitner, E. Malaguti, F. Masini, J. Puchinger, M. Ruthmair, and D. Vigo, "Overview of optimization problems in electric car-sharing system design and management," in Dynamic Perspectives on Managerial Decision Making, H. Dawid, Ed. Springer, Cham, 2016, pp. 441-471.

[67] P. S. Lavieri, V. M. Garikapati, C. R. Bhat, R. M. Pendyala, S. Astroza, and F. F. Dias, "Modeling individual preferences for ownership and sharing of autonomous vehicle technologies," Transportation Research Record: Journal of the Transportation Research Board, no. 2665, pp. 1-10, 2017.

[68] P. Jittrapirom, V. Caiati, A.-M. Feneri, S. Ebrahimigharehbaghi, M. J. Alonso González, and J. Narayan, "Mobility as a service: A critical review of definitions, assessments of schemes, and key challenges," Urban Planning, vol. 2, no. 2, pp. 13-25, 2017.

[69] R. Utriainen and M. Pöllänen, "Review on mobility as a service in scientific publications," Research in Transportation Business \& Management, 2018.

[70] M. Hao and T. Yamamoto, "Shared autonomous vehicles : A review considering car sharing and autonomous vehicles," Asian Transport Studies, vol. 5, no. 1, pp. 47-63, 2018.

[71] R. Zhang and M. Pavone, "A queueing network approach to the analysis and control of mobility-on-demand systems," in American Control Conference (ACC), 2015. IEEE, 2015, pp. 4702-4709.

[72] _ - "Control of robotic mobility-on-demand systems: A queueingtheoretical perspective," The International Journal of Robotics Research, vol. 35, no. 3, pp. 186-203, 2016.

[73] Spieser, Kevin, K. Ballantyne, Treleaven, R. Zhang, D. Morton, and M. Pavone, "Toward a systematic approach to the design and evaluation of automated mobility-on-demand systems : A case study in Singapore," in Road Vehicle Automation, Lecture Notes in Mobility,
G. Meyer and S. Beike, Eds. Springer International Publishing, 2014, ch. 20 , pp. 229-245.

[74] M. Pavone, "Autonomous mobility-on-demand systems for future urban mobility," in Autonomes Fahren. Springer, 2015, pp. 399-416.

[75] P. M. Boesch and F. Ciari, "Agent-based simulation of autonomous cars," in American Control Conference (ACC), 2015, 2015, pp. 25882592.

[76] T. D. Chen, K. M. Kockelman, and J. P. Hanna, "Operations of a shared, autonomous, electric vehicle fleet: Implications of vehicle \& charging infrastructure decisions," Transportation Research Part A: Policy and Practice, vol. 94, pp. 243-254, 2016.

[77] W. Zhang and S. Guhathakurta, "Parking spaces in the age of shared autonomous vehicles: How much parking will we need and where?" Transportation Research Record: Journal of the Transportation Research Board, no. 2651, pp. 80-91, 2017.

[78] W. Zhang, S. Guhathakurta, J. Fang, G. Zhang, W. Zhang, b. S. Guhathakurta, b. G. Zhang, S. Guhathakurta, G. Zhang, and J. Fang, "Exploring the impact of shared autonomous vehicles on urban parking demand: An agent-based simulation approach," Sustainable Cities and Society, vol. 19, pp. 34-45, 2015.

[79] A. T. Moreno, A. Michalski, C. Llorca, and R. Moeckel, "Shared autonomous vehicles effect on vehicle-km traveled and average trip duration," Journal of Advanced Transportation, vol. 0, no. 0, 2018.

[80] C. Brownell and A. Kornhauser, "A driverless alternative: Fleet size and cost requirements for a statewide Autonomous Taxi Network in New Jersey," Transportation Research Record: Journal of the Transportation Research Board, vol. 2416, pp. 73-81, 2014.

[81] F. Rossi, R. Zhang, Y. Hindy, and M. Pavone, "Routing autonomous vehicles in congested transportation networks: Structural properties and coordination algorithms," Autonomous Robots, vol. 42, no. 7, pp. 1427 $1442,2018$.

[82] R. Iglesias, F. Rossi, R. Zhang, and M. Pavone, "A bcmp network approach to modeling and controlling autonomous mobility-on-demand systems," The International Journal of Robotics Research, pp. 1-18, 2018.

[83] B. Jager, F. M. M. Agua, and M. Lienkamp, "Agent-based simulation of a shared, autonomous and electric on-demand mobility solution," in Intelligent Transportation Systems (ITSC), 2017 IEEE 20th International Conference on. IEEE, 2018, pp. 250-255.

[84] A. A. Malikopoulos, "Centralized stochastic optimal control of complex systems," in Proceedings of the 2015 European Control Conference, 2015, pp. 721-726.

[85] A. A. Malikopoulos, V. Maroulas, and J. XIong, "A multiobjective optimization framework for stochastic control of complex systems," in Proceedings of the 2015 American Control Conference, 2015, pp. 4263-4268.

[86] K. A. Marczuk, H. S. S. Hong, C. M. L. Azevedo, M. Adnan, S. D. Pendleton, E. Frazzoli, and D. H. Lee, "Autonomous mobility on demand in SimMobility: Case study of the central business district in Singapore," in Cybernetics and Intelligent Systems (CIS) and IEEE Conference on Robotics, Automation and Mechatronics (RAM), 2015 IEEE 7th International Conference on, 2015, pp. 167-172.

[87] C. L. Azevedo, K. Marczuk, S. Raveau, H. Soh, M. Adnan, K. Basak, H. Loganathan, N. Deshmunkh, D.-H. Lee, E. Frazzoli, and M. BenAkiva, "Microsimulation of demand and supply of autonomous mobility on demand," Transportation Research Record: Journal of the Transportation Research Board, vol. 2564, pp. 21-30, 2016.

[88] D. Kondor, H. Zhang, R. Tachet, P. Santi, and C. Ratti, "Estimating savings in parking demand using shared vehicles for home-work commuting," IEEE Transactions on Intelligent Transportation Systems, 2018.

[89] K. Winter, O. Cats, G. H. d. A. Correia, and B. van Arem, "Designing an Automated Demand-Responsive Transport System," Transportation Research Record: Journal of the Transportation Research Board, vol. 2542, pp. 75-83, 2016.

[90] P. M. Boesch, F. Ciari, and K. W. Axhausen, "Autonomous vehicle fleet sizes required to serve different levels of demand," Transportation Research Record: Journal of the Transportation Research Board, vol. 2542, pp. 111-119, 2016.

[91] D. J. Fagnant, K. M. Kockelman, and P. Bansal, "Operations of shared autonomous vehicle fleet for Austin, Texas, market," Transportation Research Record: Journal of the Transportation Research Board, vol. 2536, pp. 98-106, 2015.

[92] M. M. Vazifeh, P. Santi, G. Resta, S. H. Strogatz, and C. Ratti, "Addressing the minimum fleet problem in on-demand urban mobility," Nature, vol. 557, no. 7706, pp. 534-538, 2018. 
[93] N. Masoud and R. Jayakrishnan, "Autonomous or driver-less vehicles: Implementation strategies and operational concerns," Transportation Research Part E: Logistics and Transportation Review, vol. 108, pp. 179-194, 2017.

[94] M. Z. Spivey and W. B. Powell, "The dynamic assignment problem," Transportation Science, vol. 38, no. 4, pp. 399-419, 2004

[95] T. Öncan, "A survey of the generalized assignment problem and its applications," INFOR: Information Systems and Operational Research, vol. 45, no. 3, pp. 123-141, 2007.

[96] V. Pillac, M. Gendreau, C. Guéret, and A. L. Medaglia, "A review of dynamic vehicle routing problems," European Journal of Operational Research, vol. 225, no. 1, pp. 1-11, 2013.

[97] G. La porte, "Fifty years of vehicle routing," Transportation Science, vol. 43, no. 4, pp. 408-416, 2009.

[98] J. Ma, X. Li, F. Zhou, and W. Hao, "Designing optimal autonomous vehicle sharing and reservation systems: A linear programming approach," Transportation Research Part C: Emerging Technologies, vol. 84, pp. 124-141, 2017.

[99] W. Shen and C. Lopes, "Managing autonomous mobility on demand systems for better passenger experience," in Lecture Notes in Computer Science (including subseries Lecture Notes in Artificial Intelligence and Lecture Notes in Bioinformatics), 2015, vol. 9387, pp. 20-35.

[100] M. Hyland and H. S. Mahmassani, "Dynamic autonomous vehicle fleet operations: Optimization-based strategies to assign AVs to immediate traveler demand requests," Transportation Research Part C: Emerging Technologies, vol. 92, pp. 278-297, 2018.

[101] J. P. Hanna, M. Albert, D. Chen, and P. Stone, "Minimum cost matching for autonomous carsharing," IFAC PapersOnLine, vol. 49, no. 15, pp. 254-259, 2016.

[102] D. Jorge, G. H. Correia, and C. Barnhart, "Comparing optimal relocation operations with simulated relocation policies in one-way carsharing systems," IEEE Transactions on Intelligent Transportation Systems, vol. 15, no. 4, pp. 1667-1675, 2014.

[103] S. Weikl and K. Bogenberger, "Relocation strategies and algorithms for free-floating car sharing systems," IEEE Intelligent Transportation Systems Magazine, vol. 5, no. 4, pp. 100-111, 2013.

[104] A. B. Brendel, S. Lichtenberg, I. Nastjuk, and L. M. Kolbe, "Adapting carsharing vehicle relocation strategies for shared autonomous electric vehicle services," in 38th International Conference on Information Systems, Seoul, South Korea, 2017, pp. 1-20.

[105] G. G. D. Santos and G. H. de Almeida Correia, "Finding the relevance of staff-based vehicle relocations in one-way carsharing systems through the use of a simulation-based optimization tool," Journal of Intelligent Transportation Systems, pp. 1-22, 2019.

[106] R. Zhang, F. Rossi, and M. Pavone, "Model predictive control of autonomous mobility-on-demand systems," in 2016 IEEE International Conference on Robotics and Automation (ICRA). IEEE, 2016, pp. 1382-1389.

[107] J. Wen, J. Zhao, and P. Jaillet, "Rebalancing shared mobility-ondemand systems: A reinforcement learning approach," in Intelligent Transportation Systems (ITSC), 2017 IEEE 20th International Conference on. IEEE, 2017, pp. 220-225.

[108] K. Spieser, W. Gruel, and E. Frazzoli, "Shared-vehicle mobilityon-demand systems: a fleet operator's guide to rebalancing empty vehicles," in Transportation Research Board 95th Annual Meeting, 2015.

[109] M. Pavone, S. L. Smith, E. Frazzoli, and D. Rus, "Robotic load balancing for mobility-on-demand systems," The International Journal of Robotics Research, vol. 31, no. 7, pp. 839-854, 2012.

[110] S. Hörl, C. Ruch, F. Becker, E. Frazzoli, and K. W. Axhausen, "Fleet operational policies for automated mobility: A simulation assessment for zurich," Transportation Research Part C: Emerging Technologies, vol. 102, pp. 20-31, 2019.

[111] S. Zhu and A. L. Kornhauser, "The interplay between fleet size, level of service and empty vehicle repositioning strategies in largescale, shared-ride autonomous taxi mobility-on-demand scenarios," in Transportation Research Board 96th Annual Meeting, 2017, pp. 1-14.

[112] K. A. Marczuk, H. S. Soh, C. M. Azevedo, D.-H. Lee, and E. Frazzoli, "Simulation framework for rebalancing of autonomous mobility on demand systems," in MATEC Web of Conferences, M. Figueira and Z. Guo, Eds., vol. 81. EDP Sciences, 2016.

[113] K. Winter, O. Cats, B. Van Arem, and K. Martens, "Impact of relocation strategies for a fleet of shared automated vehicles on service efficiency, effectiveness and externalities," in Models and Technologies for Intelligent Transportation Systems (MT-ITS), 2017 5th IEEE International Conference on. IEEE, 2017, pp. 844-849.
[114] M. Winter, O. Cats, K. Martens, and B. van Arem, "Relocating strategies under parking constraints for a fleet of shared automated vehicles," hEART 2018, 2018.

[115] N. Kang, F. M. Feinberg, and P. Y. Papalambros, "Autonomous electric vehicle sharing system design," Journal of Mechanical Design, vol. 139, no. 1, 2016.

[116] B. Loeb, K. M. Kockelman, and J. Liu, "Shared autonomous electric vehicle (SAEV) operations across the Austin, Texas network with charging infrastructure decisions," Transportation Research Part C: Emerging Technologies, vol. 89, pp. 222-233, 2018.

[117] B. Loeb and K. M. Kockelman, "Fleet performance and cost evaluation of a shared autonomous electric vehicle (saev) fleet: A case study for austin, texas," Transportation Research Part A: Policy and Practice, vol. 121 , pp. 374 - 385, 2019.

[118] G. S. Bauer, J. B. Greenblatt, and B. F. Gerke, "Cost, energy, and environmental impact of automated electric taxi fleets in manhattan,' Environmental science \& technology, vol. 52, no. 8, pp. 4920-4928, 2018

[119] R. Iacobucci, B. McLellan, and T. Tezuka, "Modeling shared autonomous electric vehicles: Potential for transport and power grid integration," Energy, vol. 158, pp. 148-163, 2018.

[120] - "Optimization of shared autonomous electric vehicles operations with charge scheduling and vehicle-to-grid," Transportation Research Part C: Emerging Technologies, vol. 100, pp. 34-52, 2019.

[121] H. Miao, H. Jia, J. Li, and T. Z. Qiu, "Autonomous connected electric vehicle (acev)-based car-sharing system modeling and optimal planning: A unified two-stage multi-objective optimization methodology," Energy, vol. 169, pp. 797-818, 2019.

[122] V. Oldenbroek, L. A. Verhoef, and A. J. van Wijk, "Fuel cell electric vehicle as a power plant: Fully renewable integrated transport and energy system design and analysis for smart city areas," International Journal of Hydrogen Energy, vol. 42, no. 12, pp. 8166 - 8196, 2017.

[123] S. Samaranayake, K. Spieser, H. Guntha, and E. Frazzoli, "Ridepooling with trip-chaining in a shared-vehicle mobility-on-demand system," in 2017 IEEE 20th International Conference on Intelligent Transportation Systems (ITSC), 2017, pp. 1-7.

[124] M. W. Levin, K. M. Kockelman, S. D. Boyles, and T. Li, "A general framework for modeling shared autonomous vehicles with dynamic network-loading and dynamic ride-sharing application," Computers, Environment and Urban Systems, vol. 64, pp. 373-383, 2017.

[125] M. F. Hyland and H. S. Mahmassani, "Sharing is caring: Dynamic autonomous vehicle fleet operations under demand surges," in Transportation Research Board 97th Annual Meeting, 2018.

[126] D. J. Fagnant and K. M. Kockelman, "Dynamic ride-sharing and fleet sizing for a system of shared autonomous vehicles in Austin, Texas," Transportation, vol. 45, no. 1, pp. 143-158, 2018.

[127] M. Tsao, D. Milojevic, C. Ruch, M. Salazar Villalon, E. Frazzoli, and M. Pavone, "Model predictive control of ride-sharing autonomous mobility-on-demand systems," in 2016 IEEE International Conference on Robotics and Automation (ICRA), 2016, pp. 1382-1389.

[128] W. Burghout, P.-J. Rigole, and I. Andreasson, "Impacts of shared autonomous taxis in a metropolitan area," in Transportation Research Board 94th Annual Meeting, 2015.

[129] K. M. Gurumurthy and K. M. Kockelman, "Analyzing the dynamic ride-sharing potential for shared autonomous vehicle fleets using cellphone data from orlando, florida," Computers, Environment and Urban Systems, vol. 71, pp. 177 - 185, 2018.

[130] R. Iglesias, F. Rossi, K. Wang, D. Hallac, J. Leskovec, and M. Pavone, "Data-driven model predictive control of autonomous mobility-ondemand systems," in 2018 IEEE International Conference on Robotics and Automation (ICRA). IEEE, 2018, pp. 1-7.

[131] M. W. Levin, "Congestion-aware system optimal route choice for shared autonomous vehicles," Transportation Research Part C: Emerg ing Technologies, vol. 82, pp. 229-247, 2017.

[132] X. Liang, G. H. De Almeida Correia, and B. van Arem, “An optimization model for vehicle routing of automated taxi trips with dynamic travel times," Transportation Research Procedia, vol. 27, pp. 736-743, 2017.

[133] X. Liang, G. H. de Almeida Correia, and B. van Arem, "Applying a model for trip assignment and dynamic routing of automated taxis with congestion: system performance in the City of Delft, The Netherlands," Transportation Research Record, 2018.

[134] X. Liang, G. H. d. A. Correia, and B. van Arem, "Optimizing the service area and trip selection of an electric automated taxi system used for the last mile of train trips," Transportation Research Part E: Logistics and Transportation Review, vol. 93, pp. 115-129, 2016. 
[135] X. Liang, K. An, G. Homem de Almeida Correia, and B. van Arem "An optimization model of automated taxis in trip assignment under elastic demand for the first/last mile problem," hEART 2018, 2018.

[136] G. H. de Almeida Correia and B. van Arem, "Solving the user optimum privately owned automated vehicles assignment problem (uo-poavap): A model to explore the impacts of self-driving vehicles on urban mobility," Transportation Research Part B: Methodological, vol. 87, pp. 64-88, 2016.

[137] P.-J. Rigole, "Study of a shared autonomous vehicles based mobility solution in Stockholm," M.S. Thesis, Division of Industrial Ecology, Royal Institute of Technology, 2014.

[138] H. K. R. d. F. Pinto, M. F. Hyland, İ. Ö. Verbas, H. S. Mahmassani, and T. R. Board, "Integrated mode choice and dynamic traveler assignmentsimulation framework to assess the impact of a suburban first-mile shared autonomous vehicle fleet service on transit demand," Transportation Research Record: Journal of the Transportation Research Board, 2018

[139] D. Fiedler, M. Cap, and M. Certicky, "Impact of mobility-on-demand on traffic congestion: Simulation-based study," in Intelligent Transportation Systems (ITSC), 2017 IEEE 20th International Conference on. IEEE, 2018, pp. 1-6.

[140] A. Scheltes and G. H. de Almeida Correia, "Exploring the use of automated vehicles as last mile connection of train trips through an agent-based simulation model: An application to Delft, Netherlands," International Journal of Transportation Science and Technology, vol. 6 , no. 1, pp. 28-41, 2017.

[141] J. Farhan and T. D. Chen, "Impact of ridesharing on operational efficiency of shared autonomous electric vehicle fleet," Transportation Research Part C: Emerging Technologies, vol. 93, pp. 310-321, 2018.

[142] W. Zhang, S. Guhathakurta, J. Fang, and G. Zhang, "The performance and benefits of a shared autonomous vehicles based dynamic ridesharing system : An agent-based simulation approach," in Transportation Research Board 94th Annual Meeting, 2015.

[143] J. Zachariah, J. Gao, A. Kornhauser, and T. Mufti, "Uncongested mobility for all: A proposal for an area wide autonomous taxi system in New Jersey," in Transportation Research Board 93rd Annual Meeting, 2014, pp. 1-14.

[144] M. Lu, M. Taiebat, M. Xu, and S.-C. Hsu, "Multiagent spatial simulation of autonomous taxis for urban commute: Travel economics and environmental impacts," Journal of Urban Planning and Development, vol. 144, no. 4, 2018.

[145] W. Shen, C. V. Lopes, and J. W. Crandall, "An online mechanism for ridesharing in autonomous mobility-on-demand systems," in TwentyFifth International Joint Conference on Artificial Intelligence (IJCAI16), 2016, pp. 475-481.

[146] Y. Shen, H. Zhang, and J. Zhao, "Integrating shared autonomous vehicle in public transportation system: A supply-side simulation of the first-mile service in singapore," Transportation Research Part A: Policy and Practice, vol. 113, pp. 125-136, 2018.

[147] R. Chen and C. G. Cassandras, "Optimization of ride sharing systems using event-driven receding horizon control," arXiv preprint arXiv:1901.01919, 2019

[148] S. Hörl, "Implementation of an autonomous taxi service in a multimodal traffic simulation using MATSim," Ph.D. dissertation, Chalmers University of Technology, 2016.

[149] J. Liu, K. M. Kockelman, P. M. Boesch, and F. Ciari, "Tracking a system of shared autonomous vehicles across the Austin, Texas network using agent-based simulation," Transportation, vol. 44, no. 6, pp. 12611278, 2017.

[150] L. M. Martinez and J. M. Viegas, "Assessing the impacts of deploying a shared self-driving urban mobility system: An agent-based model applied to the city of Lisbon, Portugal," International Journal of Transportation Science and Technology, vol. 6, no. 1, pp. 13-27, 2017.

[151] Y. Shen, H. Zhang, and J. Zhao, "Embedding autonomous vehicle sharing in a public transit system: Example of last-mile problem," in Transportation Research Board 96th Annual Meeting, 2017.

[152] M. Snelder, I. Wilmink, J. van der Gun, H. J. Bergveld, P. Hoseini, and B. van Arem, "Mobility impacts of automated driving and shared mobility-explorative model and case study of the province of northholland," in Transportation Research Board 98th Annual Meeting, 2019.

[153] J. Wen, Y. X. Chen, N. Nassir, and J. Zhao, "Transit-oriented autonomous vehicle operation with integrated demand-supply interaction," Transportation Research Part C: Emerging Technologies, vol. 97, pp. 216-234, 2018.

[154] R. Basu, A. Araldo, A. P. Akkinepally, B. H. N. Biran, K. Basak, R. Seshadri, N. Deshmukh, N. Kumar, C. L. Azevedo, and M. Ben-
Akiva, "Automated mobility-on-demand vs. mass transit: A multimodal activity-driven agent-based simulation approach," Transportation Research Record, vol. 0, no. 0, 2018.

[155] R. Lamotte, A. De Palma, and N. Geroliminis, "On the use of reservation-based autonomous vehicles for demand management," Transportation Research Part B: Methodological, vol. 99, pp. 205-227, 2017.

[156] M. Maciejewski and J. Bischoff, "Congestion effects of autonomous taxi fleets," Transport, pp. 1-10, 2017.

[157] B. Remer and A. A. Malikopoulos, "The multi-objective dynamic traveling salesman problem: Last mile delivery with unmanned aerial vehicles assistance," in American Control Conference, 2019 (to appear).

[158] R. Boersma, B. van Arem, and F. Rieck, "Application of driverless electric automated shuttles for public transport in villages: The case of appelscha," World Electric vehicle journal, vol. 9, no. 1, p. 15, 2018.

[159] Z. Wadud, D. MacKenzie, and P. Leiby, "Help or hindrance? the travel, energy and carbon impacts of highly automated vehicles," Transportation Research Part A: Policy and Practice, vol. 86, pp. 1-18, 2016.

[160] D. Milakis, B. Van Arem, and B. Van Wee, "Policy and society related implications of automated driving: A review of literature and directions for future research," Journal of Intelligent Transportation Systems, vol. 21, no. 4, pp. 324-348, 2017.

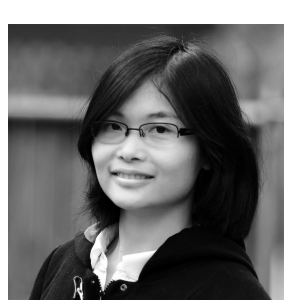

Liuhui Zhao (M2017) received the B.S. degree in Resources Science and Technology from Beijing Normal University, Beijing, China, in 2009, the M.S. degree from Department of Geography at the University of Alabama in 2011, and the Ph.D. degree in Transportation Engineering from New Jersey Institute of Technology in 2016. She is currently a Postdoctoral Researcher in the Information and Decision Science (IDS) Laboratory at the University of Delaware leading research projects on emerging transportation systems. She has participated in various research projects on connected automated vehicles, intelligent transportation systems, traffic and transit operations. Her research interests lie within the areas of intelligent transportation systems, shared mobility, and connected automated vehicles.

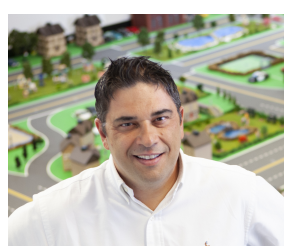

Andreas A. Malikopoulos (M2006, SM2017) received a Diploma in Mechanical Engineering from the National Technical University of Athens, Greece, in 2000. He received M.S. and Ph.D. degrees from the Department of Mechanical Engineering at the University of Michigan, Ann Arbor, Michigan, USA, in 2004 and 2008, respectively. He is the Terri Connor Kelly and John Kelly Career Development Associate Professor in the Department of Mechanical Engineering at the University of Delaware (UD) and the Director of the Information and Decision Science (IDS) Laboratory. Before he joined UD, he was the Deputy Director and the Lead of the Sustainable Mobility Theme of the Urban Dynamics Institute at Oak Ridge National Laboratory, and a Senior Researcher with General Motors Global Research \& Development. His research spans several fields, including analysis, optimization, and control of cyber-physical systems; decentralized systems; and stochastic scheduling and resource allocation problems. The emphasis is on applications related to sociotechnical systems, energy efficient mobility systems, and sustainable systems. He is currently an Associate Editor of the IEEE Transactions on Intelligent Vehicles and IEEE Transactions on Intelligent Transportation Systems. He is a member of SIAM, AAAS, and a Fellow of the ASME. 